\title{
Restoration of interferometric images
}

\section{Efficient Richardson-Lucy methods for LINC-NIRVANA data reduction}

\author{
B. Anconelli ${ }^{1}$, M. Bertero ${ }^{1}$, P. Boccacci $^{1}$, M. Carbillet $^{2}$, and H. Lanteri ${ }^{2}$ \\ 1 INFM and DISI, Università di Genova, via Dodecaneso 35, 16146 Genova, Italy \\ e-mail: bertero@disi.unige.it \\ ${ }^{2}$ Laboratoire Universitaire d'Astrophysique de Nice, UMR 6525, Parc Valrose, 06108 Nice Cedex 02, France
}

Received 18 June 2004 / Accepted 7 September 2004

\begin{abstract}
In previous papers we proposed methods and software for the restoration of images provided by Fizeau interferometers such as LINC-NIRVANA (LN), the German-Italian beam combiner for the Large Binocular Telescope (LBT). It will provide multiple images of the same target corresponding to different orientations of the baseline. Therefore LN will require routinely the use of multiple-image deconvolution methods in order to produce a unique high-resolution image. As a consequence of the complexity of astronomical images, two kinds of methods will be required: first a quick-look method, namely a method that is computationally efficient, allowing a rapid overview and identification of the object being observed; second an ad hoc method designed for that particular object and as accurate as possible. In this paper we investigate the possibility of using Richardson-Lucy-like (RL-like) methods, namely methods designed for the maximization of the likelihood function in the case of Poisson noise, as possible quick-look methods. To this purpose we propose new techniques for accelerating the Ordered Subsets - Expectation Maximization (OS-EM) method, investigated in our previous papers; moreover, we analyze approaches based on the fusion of the multiple images into a single one, so that one can use single-image deconvolution methods which are presumably more efficient than the multiple-image ones. The results are encouraging and all the methods proposed in this paper have been implemented in our software package AIRY.
\end{abstract}

Key words. techniques: image processing - techniques: interferometric

\section{Introduction}

In previous papers (Bertero \& Boccacci 2000a,b; Correia et al. 2002; Carbillet et al. 2002) we developed methods and software for the deconvolution of multiple interferometric images of the same astronomical target. Moreover, our group has produced the software package AIRY (Astronomical Image Reconstruction in interferometrY) of which version 2.0 is now available (see http://dirac.disi.unige.it and http://www.arcetri.astro.it/caos). This tool can be applied to Fizeau interferometers such as the beam combiner for LBT, denoted as LINC-NIRVANA (Lbt INterferometric Camera and Near-InfraRed/Visible Adaptive iNterferometer for Astronomy). LBT will consist of two $8.4 \mathrm{~m}$ mirrors on a common mount, with a spacing of $14.4 \mathrm{~m}$ between their centres, so that a maximum baseline of $22.8 \mathrm{~m}$ will be available. First light of the telescope is scheduled for September 2004, second light for November 2005 while the first interferometric light is foreseen for mid 2006.

The interferometric technique used in $\mathrm{LN}$ will provide direct imaging with the resolution of a $22.8 \mathrm{~m}$ telescope in the direction of the baseline and of a $8.4 \mathrm{~m}$ telescope in the orthogonal direction. Since resolution is not uniform over the field, several images of the same scientific object must be acquired with different orientations of the baseline and they must be routinely processed (deconvolved) to get a unique image with the resolution of a $22.8 \mathrm{~m}$ telescope.

Two different kinds of deconvolution methods may be needed:

- quick-look methods, computationally efficient even if not always very accurate, to be routinely used for a preliminary view of the target just after data acquisition;

- ad hoc methods, designed for specific classes of astronomical objects, which can be computationally expensive but must be accurate as far as possible for all the objects in a given class.

Therefore the processing of $\mathrm{LN}$ images will in general require two steps: the first, based on a quick-look method, is intended to identify the specific features of the particular object the astronomer is observing; the second consists in the use of the ad hoc method which has been designed for the objects with those specific features.

An example of a quick-look method is provided by the so-called Tikhonov regularization (Bertero \& Boccacci 1998, 2000a), which is basically a Wiener filter. More accurate, even if less efficient, is the OS-EM method, proposed by 
Hudson \& Larkin (1994) for emission tomography and extended to LBT-imaging in Bertero \& Boccacci (2000b), which provides the same accuracy as the RL method (Richardson 1972; Lucy 1974), but with a considerable reduction in computational cost. On the other hand ad hoc methods could be designed for specific objects such as objects with a very high dynamic range (an example is the model of a young binary proposed by Carbillet et al. 2002), or unresolved compact objects (an example is the method, proposed by Correia et al. (2002) and validated by Anconelli et al. (2005), for super-resolving binary systems) or others.

In any case computational efficiency is important for the processing of LN images. The first detector will cover a field of about $10^{\prime \prime}$ in $K$ band, with a pixel size of about 5 mas, so that it will produce images of about $2000 \times 2000$ pixels. The processing of these images is not a terrible task. But, since the AO-system of LN provides a uniform correction over a field of $2^{\prime}$, it is reasonable to expect that even broader images will be available in the future. For this reason we are interested in increasing the efficiency of RL-like algorithms.

We consider two approaches: the first consists in improving the efficiency of the OS-EM algorithm by means of a technique which allows a reduction of the number of iterations, without a significant increase of the computational cost of a single iteration; the second consists in the combination of the multi-images provided by LN into a single one. One of these approaches has been recently proposed by Vio et al. (2004). It is obvious that, in such a case, the computational cost of one iteration is that of a single image. However this is true also for the OS-EM method so that the comparison of the efficiency of all these methods is uniquely based on the comparison of the number of iterations required for getting the optimal restoration.

In Sect. 2 we propose our method for OS-EM acceleration while in Sect. 3 we analyze a number of single-image approaches. In Sect. 4 we present numerical experiments intended to both evaluate possible acceleration factors and to compare the efficiency and accuracy of multi-image and single-image methods. The results are encouraging, as we discuss in Sect. 5.

\section{The accelerated version of the OS-EM algorithm}

In this paper $N \times N$ arrays are denoted by bold letters. Let us assume that we have $p$ images acquired with $\mathrm{LN}$, corresponding to $p$ different orientations of the baseline and denoted by $\boldsymbol{g}_{1}, \boldsymbol{g}_{2}, \ldots, \boldsymbol{g}_{p}$. Then, if $\boldsymbol{g}_{j}(m, n),(j=1, \ldots, p)$ is the value of one of these images at pixel $m, n$, according to the model proposed by Snyder et al. (1993) for images acquired with a CCD camera, we can write:

$\boldsymbol{g}_{j}(m, n)=\boldsymbol{g}_{\mathrm{obj}, j}(m, n)+\boldsymbol{g}_{\mathrm{back}, j}(m, n)+\boldsymbol{r}_{j}(m, n)$,

where: $\boldsymbol{g}_{\mathrm{obj}, j}(m, n)$ is the number of photoelectrons due to radiation from the object; $\boldsymbol{g}_{\text {back }, j}(m, n)$ is the number of photoelectrons due to external and internal background, dark current, etc.; $\boldsymbol{r}_{j}(m, n)$ is the read-out noise due to the amplifier. The first two terms are realizations of independent Poisson processes (photon noise), so that their sum is also a Poisson process and its expected value is given by:

$$
\begin{aligned}
E\left\{\boldsymbol{g}_{\mathrm{obj}, j}(m, n)+\right. & \left.\boldsymbol{g}_{\mathrm{back}, j}(m, n)\right\}= \\
& \left(\boldsymbol{K}_{j} * \boldsymbol{f}\right)(m, n)+\boldsymbol{b}_{j}(m, n),
\end{aligned}
$$

where: $\boldsymbol{K}_{j}$ is the point spread function (PSF), corresponding to the $j$ th orientation of the baseline (we assume, for simplicity, space invariance); $\boldsymbol{f}$ is the object array, formed by the average numbers of photons emitted at the pixels of the object domain and collected by the telescope; $\boldsymbol{b}_{j}(m, n)$ is the expected value of the background. In the following we will denote by $A_{j}$ the block-circulant matrix which is defined by the convolution product with the PSF $\boldsymbol{K}_{j}$ :

$A_{j} \boldsymbol{f}=\boldsymbol{K}_{j} * f$,

and by $A_{j}^{T}$ the transposed matrix. Moreover we will assume that the PSFs are normalized in such a way that the sum of their pixel values is one.

Finally the last term in Eq. (1) is the realization of an independent Gaussian process with expected value $r$ and variance $\sigma^{2}$ (white noise). In the software package AIRY, which is described by Correia et al. (2002) and is used for our numerical simulations, images are generated according to this model.

In the case of images dominated by photon noise, the readout noise can be neglected, and the likelihood function is given by a product of Poisson distributions, one for each pixel of the image domain. The maximization of this function is equivalent to the minimization of the Csiszár directed divergence (Csiszár 1991) given by:

$$
\begin{aligned}
J(\boldsymbol{f})= & \sum_{j=1}^{p} \sum_{m, n=0}^{N-1}\left\{\boldsymbol{g}_{j}(m, n) \ln \frac{\boldsymbol{g}_{j}(m, n)}{\left(A_{j} \boldsymbol{f}\right)(m, n)+\boldsymbol{b}_{j}(m, n)}\right. \\
& \left.+\left[\left(A_{j} \boldsymbol{f}\right)(m, n)+\boldsymbol{b}_{j}-\boldsymbol{g}_{j}(m, n)\right]\right\} .
\end{aligned}
$$

The new version of OS-EM can be introduced and justified in the framework of a general approach, proposed by Lanteri et al. (2001), which makes it possible to design descent methods for the minimization of a convex functional $J(f)$ such as that of Eq. (4). The basic idea relies on the following decomposition of the gradient of the functional:

$-\nabla J(f)=U(f)-V(f)$,

where $\boldsymbol{U}(\boldsymbol{f})$ and $\boldsymbol{V}(\boldsymbol{f})$ are positive arrays. Such a decomposition always exists and is not unique; the applicability of the method requires an explicit expression for the dependence of these arrays on $f$. Then the general structure of the proposed iterative algorithms is as follows:

$\boldsymbol{f}^{(k+1)}=\boldsymbol{f}^{(k)}+\alpha \boldsymbol{f}^{(k)} \frac{\boldsymbol{U}\left(\boldsymbol{f}^{(k)}\right)^{\omega}-\boldsymbol{V}\left(\boldsymbol{f}^{(k)}\right)^{\omega}}{\boldsymbol{V}\left(\boldsymbol{f}^{(k)}\right)^{\omega}}$,

where the quotient of two arrays is defined pixel by pixel as we will do also in the next equations. $\alpha$ and $\omega$ are relaxation parameters. $\alpha$ is the step-size in the descent direction (modified by $\omega$ if $\omega>1$ ) and it can be chosen to guarantee both the nonnegativity of the iterates and their convergence. On the other hand $\omega$ is a parameter that can allow a reduction of the number of iterations, as we will discuss below. We will call it the acceleration exponent. 
Indeed, as discussed by Lanteri et al. (2001), an integer value of $\omega>1$ can speed up the convergence of the iterations. At the very first iterations $\omega$ modifies the descent direction; but, when the iterations are close to convergence, the algorithm with $\omega>1$ is approximately equivalent to the algorithm with $\omega=1$ and step-size $\alpha \omega$. For the convenience of the reader we give the argument in the simple case $\omega=2$. If we write Eq. (6) in the following form:

$$
\begin{aligned}
\boldsymbol{f}^{(k+1)}= & \boldsymbol{f}^{(k)}+\alpha \boldsymbol{f}^{(k)} \frac{\boldsymbol{U}\left(\boldsymbol{f}^{(k)}\right)+\boldsymbol{V}\left(\boldsymbol{f}^{(k)}\right)}{\boldsymbol{V}\left(\boldsymbol{f}^{(k)}\right)^{2}} \\
& \times\left[\boldsymbol{U}\left(\boldsymbol{f}^{(k)}\right)-\boldsymbol{V}\left(\boldsymbol{f}^{(k)}\right)\right],
\end{aligned}
$$

and if we observe that $\nabla J(f) \simeq 0$ when we are close to convergence, so that $\boldsymbol{U}\left(\boldsymbol{f}^{(k)}\right) \simeq \boldsymbol{V}\left(\boldsymbol{f}^{(k)}\right)$, then we get:

$\boldsymbol{f}^{(k+1)} \simeq \boldsymbol{f}^{(k)}+2 \alpha \boldsymbol{f}^{(k)} \frac{\boldsymbol{U}\left(\boldsymbol{f}^{(k)}\right)-\boldsymbol{V}\left(\boldsymbol{f}^{(k)}\right)}{\boldsymbol{V}\left(\boldsymbol{f}^{(k)}\right)}$,

i.e. the algorithm of Eq. (6) with $\omega=1$ and step-size $2 \alpha$.

In the case of the functional of Eq. (4) we get:

$$
\begin{aligned}
\boldsymbol{U}(\boldsymbol{f}) & =\sum_{j=1}^{p} A_{j}^{T} \frac{\boldsymbol{g}_{j}}{A_{j} \boldsymbol{f}+\boldsymbol{b}_{j}}, \\
\boldsymbol{V}(\boldsymbol{f}) & =\sum_{j=1}^{p} A_{j}^{T} \mathbf{1}=p \mathbf{1},
\end{aligned}
$$

where $\mathbf{1}$ is the array whose entries are all equal to 1 . If we insert these equations in Eq. (6) we obtain a number of known algorithms. For instance, if $\omega=1$ and $\alpha=1$, we obtain just the RL method for multiple-image deconvolution given in Bertero \& Boccacci (2000b). On the other hand, if $\omega=1$ and $\alpha \neq 1$, we obtain the extension to the multiple-image case of the relaxed RL-method investigated by several authors (Kosarev et al. 1983; Holmes \& Liu 1991; Adorf et al. 1993). As is known, this method provides a considerable reduction of the number of iterations but with an increase of the computational cost of each iteration, due to the search for the value of $\alpha$. For example, if the step-size $\alpha$ is computed by means of an approximate search method, such as the Armijo rule, instead of the exact one (this is possible in our case), the average reduction of the overall computation time is about 20\% (Lanteri et al. 2001), depending on the images considered and on the noise level.

Finally, in the case $\alpha=1$ and $\omega>1$, we obtain the extension to the multiple-image case of an algorithm proposed by Llacer \& Nuñez (1990):

$\boldsymbol{f}^{(k+1)}=\boldsymbol{f}^{(k)}\left(\frac{1}{p} \sum_{j=1}^{p} A_{j}^{T} \frac{\boldsymbol{g}_{j}}{A_{j} \boldsymbol{f}^{(k)}+\boldsymbol{b}_{j}}\right)^{\omega}$.

The convergence of this algorithm is not proved and therefore it must be investigated theoretically and/or experimentally. As shown by Natterer \& Wübbeling (2001), convergence presumably holds true when $\omega$ is not too large. It is obvious that the most interesting feature of the algorithm is that, as in the case of RL, non-negativity of the iterates is automatically assured, as one can easily verify. Moreover, in the case of tomography, it is quite natural to extend the trick to OS-EM itself (see
Tanaka 1987; Natterer \& Wübbeling 2001), and what we propose in this paper is just the extension of this algorithm to multiple-image deconvolution.

However, there is an important point which must be taken into account. In the case of zero background, as shown by Bertero \& Boccacci (2000a), the total flux of each iterate of the multi-image RL method coincides with the arithmetic mean of the total fluxes of the images $\boldsymbol{g}_{j}$. As a consequence of the introduction of the background terms, the iterates of the RL and OS-EM methods do not have this property. Therefore, it is convenient to require that the total flux of the solution coincides with the arithmetic mean of the total fluxes of the images $g_{j}$, after subtraction of the background terms (which are not due to the scientific object):

$$
\begin{aligned}
\sum_{m, n=0}^{N-1} \boldsymbol{f}(m, n) & =c \\
& =\frac{1}{p} \sum_{j=1}^{p} \sum_{m, n=0}^{N-1}\left\{\boldsymbol{g}_{j}(m, n)-\boldsymbol{b}_{j}(m, n)\right\} .
\end{aligned}
$$

As shown by Lanteri et al. (2002), to minimize the functional of Eq. (4) with this constraint is equivalent to normalizing, at each step, the iterates in such a way that they satisfy this condition. In the case of the accelerated algorithm we have verified that this normalization is necessary to assure convergence.

In conclusion, the algorithm we have implemented in AIRY and tested is as follows:

- compute the constant $c$, as defined in Eq. (11);

- choose an initial $\boldsymbol{f}^{(0)} \geq 0$ such that its total flux coincides with $c$;

- given $\boldsymbol{f}^{(k)}$, set $j=(k+1) \bmod p$ and compute:

$$
\begin{aligned}
\tilde{\boldsymbol{f}}^{(k+1)} & =\boldsymbol{f}^{(k)}\left(A_{j}^{T} \frac{\boldsymbol{g}_{j}}{A_{j} \boldsymbol{f}^{(k)}+\boldsymbol{b}_{j}}\right)^{\omega}, \\
\tilde{c}^{(k+1)} & =\sum_{m, n=0}^{N-1} \tilde{\boldsymbol{f}}^{(k+\mathbf{1})}(m, n) ;
\end{aligned}
$$

- set:

$$
\boldsymbol{f}^{(k+1)}=\frac{c}{\tilde{c}^{(k+1)}} \tilde{\boldsymbol{f}}^{(k+1)} .
$$

For $\omega=1$ we obtain the standard OS-EM method. However, we point out that the OS-EM iterations are defined in a different way in Bertero \& Boccacci (2000a) and in the subsequent papers: in those papers one iteration consists of a cycle over the $p$ images, so that it consists of $p$ iterations of the algorithm defined above. The new version makes easy the comparison with the single-image approaches discussed in the next section. Indeed, it is evident that the computational cost of one iteration is just that of a single-image iteration; the only difference is that the image changes at each step.

We recall that, as proved in our previous papers, the computational gain of OS-EM with respect to standard multi-image $\mathrm{RL}$ is of the order of $4 /(3 p+1)$. In the probably frequent case of 3 images, the computation time of OS-EM is about $40 \%$ of that of RL, while for 4 images it is about $30 \%$. A further reduction is provided by the algorithm of Eq. (13). Indeed, as a result 
of many numerical experiments we can state that, for $\omega=2$, the method is convergent and always provides a reduction of the number of iterations by a factor 2 , hence a reduction by a factor 2 of the computational cost, which thus is of the order of (at least) $20 \%$ of that of standard RL. Moreover, numerical experiments described in Sect. 4 indicate that it is possible to increase the acceleration exponent in a way that depends on the signal-to-noise ratio (SNR): smaller SNR may be compatible with a higher acceleration exponent. As we will show, an exponent of 8 is possible in some cases.

We conclude by remarking that $\omega$ does not need to be integer, as pointed out by Tanaka (1987), so that one can also investigate non-stationary methods, namely methods where $\omega$ changes at each iteration.

\section{Single-image approaches}

In a recent paper Vio et al. (2004) propose to solve the multiimage deconvolution problem by combining the different images into a single one containing all relevant information. At first glance such an approach looks appealing: first because it becomes possible to use existing software for image deconvolution and secondly because the computational cost seems to be reduced. However, we recall that, for an algorithm such as OS-EM, the cost of one iteration is just that of one single-image RL-iteration. Moreover two other points should not be forgotten: first, in the case of imaging systems such as the LBT interferometer where the AO correction, the angular smearing (see next section), and the time exposure (and hence the noise contributions and the resulting background) can be different for each different baseline exposure, the link between the (very) different PSFs and the corresponding images may be lost when the multi-images are combined into a single one and this may result in a loss of relevant information; secondly, the statistical properties of the noise may be modified by the fusion process. Hence, the efficiency and accuracy of the single-image approaches must be tested numerically.

There are many ways of combining the multi-images into a single one. In this section we consider two of them. In all cases we assume that, if different images correspond to different integration times, $\tau_{1}, \tau_{2}, \ldots, \tau_{p}$ then all images have been normalized, for instance, to the largest integration time $\tau_{\max }$, i.e. $\boldsymbol{g}_{j}$ has been multiplied by $\tau_{\max } / \tau_{j}$.

\section{Method 1}

The first approach is the most natural one and consists in a simple addition of the different images. In order to preserve normalization of the PSF it is convenient to take the arithmetic mean. Therefore the PSF will be given by:

$$
\boldsymbol{K}_{A V}(m, n)=\frac{1}{p} \sum_{j=1}^{p} \boldsymbol{K}_{j}(m, n),
$$

with the corresponding image

$$
\boldsymbol{g}_{A V}(m, n)=\frac{1}{p} \sum_{j=1}^{p} \boldsymbol{g}_{j}(m, n) .
$$
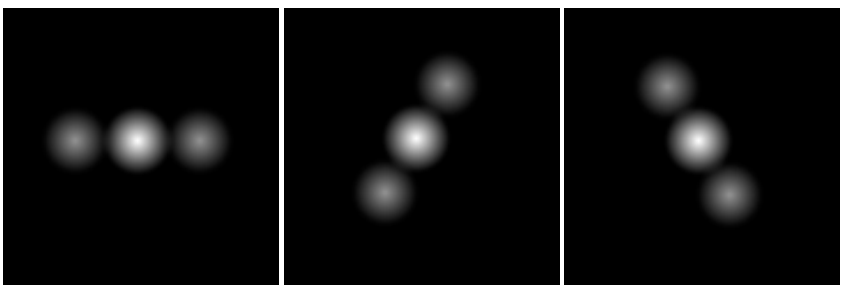

Fig. 1. Examples of MTFs corresponding to three orientations of the LBT baseline (ideal case): $0^{\circ}$ in the left panel, $60^{\circ}$ in the central panel and $120^{\circ}$ in the right panel.
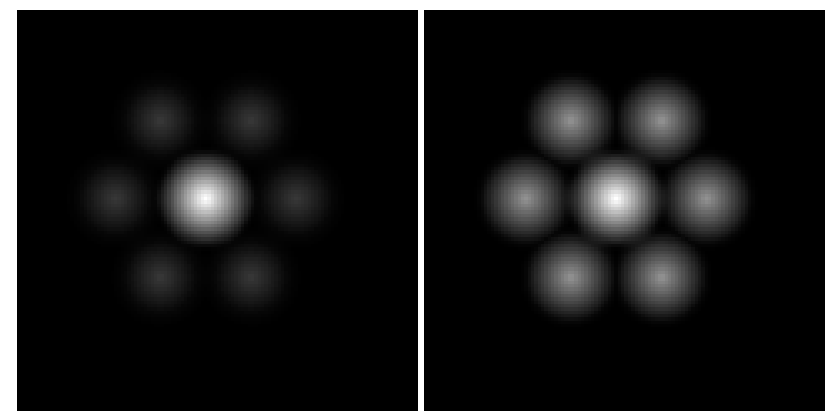

Fig. 2. MTFs of the single-image approaches, corresponding to the ideal MTFs of Fig. 1: method 1 in the left panel and method 2 in the right panel.

The main features of this approach are the following:

- Both the PSF and the image are non-negative; moreover the PSF is correctly normalized to 1 and the background of the image $\boldsymbol{g}_{A V}$ is the arithmetic mean of the backgrounds of the images $\boldsymbol{g}_{j}$.

- If the images $\boldsymbol{g}_{j}$ are corrupted by Poisson (photon) noise, then their sum is also corrupted by Poisson noise, since the sum of independent Poisson processes is also a Poisson process with an expected value given by the sum of the expected values.

- The addition of the images implies that in the domains of the $u, v$ plane where only one image contains information, the effect of the others is just to add noise, hence to reduce the SNR.

Figure 1 can help to clarify the last statement. We assume three ideal PSFs corresponding to three different parallactic angles: $0^{\circ}, 60^{\circ}$, and $120^{\circ}$ respectively, and we plot the MTFs (Modulation Transfer Functions) of these PSFs. It is obvious that all the Fourier transforms of the corresponding images will take approximately the same values in the central disc; these values are added together so that the signal will be the arithmetic mean of the signals coming from the three images. On the other hand, in the secondary discs only one image will contribute with significant values, while the other two will add noise, producing a degradation of the signal. The first picture to the left in Fig. 2 is the MTF of the average PSF, $\boldsymbol{K}_{A V}$, in the case of the ideal PSFs of Fig. 1

\section{Method 2}

In a recent paper Vio et al. (2004) propose a new method, derived from the least-squares approach, for the fusion of the 
multiple images into a single one. However, the relationship with the least-squares approach is not relevant and, in our paper, we consider a different kind of fusion which does not coincide exactly with that proposed by Vio et al. (2004) and has simpler properties.

The basic idea of the approach of Vio et al. is to define a transfer function $\hat{\boldsymbol{K}}_{M M}(m, n)$ which is obtained by taking, in a pixel $m, n$ of the $u, v$ plane, the value of the transfer function which has the maximum modulus, i.e.

$$
\begin{aligned}
\hat{\boldsymbol{K}}_{M M}(m, n) & =\hat{\boldsymbol{K}}_{J}(m, n), \text { if } \\
\left|\hat{\boldsymbol{K}}_{J}(m, n)\right| & \geq\left|\hat{\boldsymbol{K}}_{j}(m, n)\right|, \text { for } j=1, \ldots, p .
\end{aligned}
$$

The MTF of this method is given in the right panel of Fig. 2, also for the ideal MTFs of Fig. 1. As is obvious, this procedure provides the correct contrast between the central disc and the secondary discs, since these reproduce correctly those of the original MTFs.

Then we introduce the image which is quite naturally related to the transfer function defined in Eq. (17): it is defined by taking, in a pixel $m, n$ of the $u, v$ plane, just the value of the Fourier transform of the image with index $J$, i.e. that corresponding to the transfer function with the maximum modulus. We have:

$\hat{\boldsymbol{g}}_{M M}(m, n)=\hat{\boldsymbol{g}}_{J}(m, n)$.

The main features of this approach are the following:

- Both the PSF and the image, defined as the inverse Fourier transforms of Eqs. (17) and (18) respectively, may take negative values. These must be set to zero if the RL method is used. Therefore the PSF must be renormalized to guarantee that the sum of the pixel values is 1 , and the total flux of the non-negative image must be computed. For simplicity we do not change the notations of the PSF and of the image obtained from the original ones through these operations.

- The background of the image $\boldsymbol{g}_{M M}$ will coincide with the largest background in the detected images, a difficulty in the case of very different observation times, so that renormalization of the images mentioned at the beginning of this section is required.

- In the case of white noise affecting the images $\boldsymbol{g}_{j}$, the noise affecting the image $\boldsymbol{g}_{M M}$ of Eq. (18) has a constant power spectrum.

If we apply the RL-algorithm to the images of the two methods described above, it is obvious that the computational cost of one iteration is precisely that of one OS-EM iteration. Moreover, it is obvious that the same acceleration exponent can be applied in all cases. Therefore the comparison between the efficiencies of the three methods simply consists in the comparison between the numbers of iterations required for reaching the optimal restorations. Since the optimal restorations provided by the three methods, in general, are not the same, comparing them gives insight in their accuracy.

\section{Numerical experiments}

The purpose of our numerical experiments is twofold:

- investigate possible criteria for deciding the maximum value of the acceleration exponent $\omega$ which is compatible with the convergence of the iterative algorithm;

- compare the accuracy and efficiency of the accelerated multi-image algorithm of Eq. (13) with $\omega=2$, and that of the accelerated single-image RL algorithm (same equations with $p=1$ ) applied to the images provided by method 1 and method 2 of the previous section.

We will denote the three approaches respectively as OS-EM, RL-AV and RL-MM.

We first describe the three sets of PSFs used in our experiments. All the sets correspond to orientations of the baseline with respect to the observed object (relative parallactic angles) of $0^{\circ}, 60^{\circ}$ and $120^{\circ}$. The first set consists of ideal PSFs, the second of AO-corrected PSFs with different Strehl ratios for the three different orientations, and the third of AO-corrected PSFs with the same Strehl ratios as before but with the addition of an angular smearing effect due to earth rotation during the observation (which depends also on the declination of the object - see Carbillet et al. 2002).

The AO-correction effects are obtained, as already described in Carbillet et al. (2002), by an accurate model and simulation of the actual AO system aboard LBT by means of the software package CAOS (Carbillet et al. 2004). The atmospheric conditions assumed result in a multi-layer turbulent atmosphere whose main physical parameters are: a mean velocity of $15 \mathrm{~m} / \mathrm{s}$, a wave-front outer scale of $20 \mathrm{~m}$, and values of the total Fried parameter of 12,18 , and $15 \mathrm{~cm}$ (at $500 \mathrm{~nm}$ ), respectively, for the PSF at $0^{\circ}, 60^{\circ}$, and $120^{\circ}$; these clearly correspond to three different moments of observation during the night. As concerns the AO system itself, which is pyramid-based, the physical parameters are those foreseen for the system of LBT. We assume a configuration of $15 \times 15$ equivalent sub-apertures, a $13^{\text {th }}$ magnitude guide star with optimized exposure time and a number of LBT 672 mirror modes for each of the three different atmospheric conditions. The resulting Strehl ratios are 52\% for the PSF at $0^{\circ}, 87 \%$ for the PSF at $60^{\circ}$, and $79 \%$ for the PSF at $120^{\circ}$.

In the third set the first and the third PSFs are integrated over an angle of about $5^{\circ}$, while the second is integrated over an angle of about $45^{\circ}$; in practice this angular smearing effect is obtained by adding snapshot PSFs for different orientations. The two sets of AO-corrected PSFs are shown in Fig. 3.

For the objects, we consider a series of diffuse objects and a pointlike one. These objects are relatively simple, but are suitable if our purpose is mainly to compare efficiency and accuracy of the different methods.

In a first set of numerical experiments, intended to estimate the maximum value of the acceleration exponent which is compatible with the convergence of the algorithm, we use eleven different nebulae observed with the Hubble Space Telescope and moved far out in order to match an angular resolution relevant for the $22.8 \mathrm{~m}$ equivalent aperture of LBT. For example the young stellar object (YSO) IRAS 04302+2247, which is 


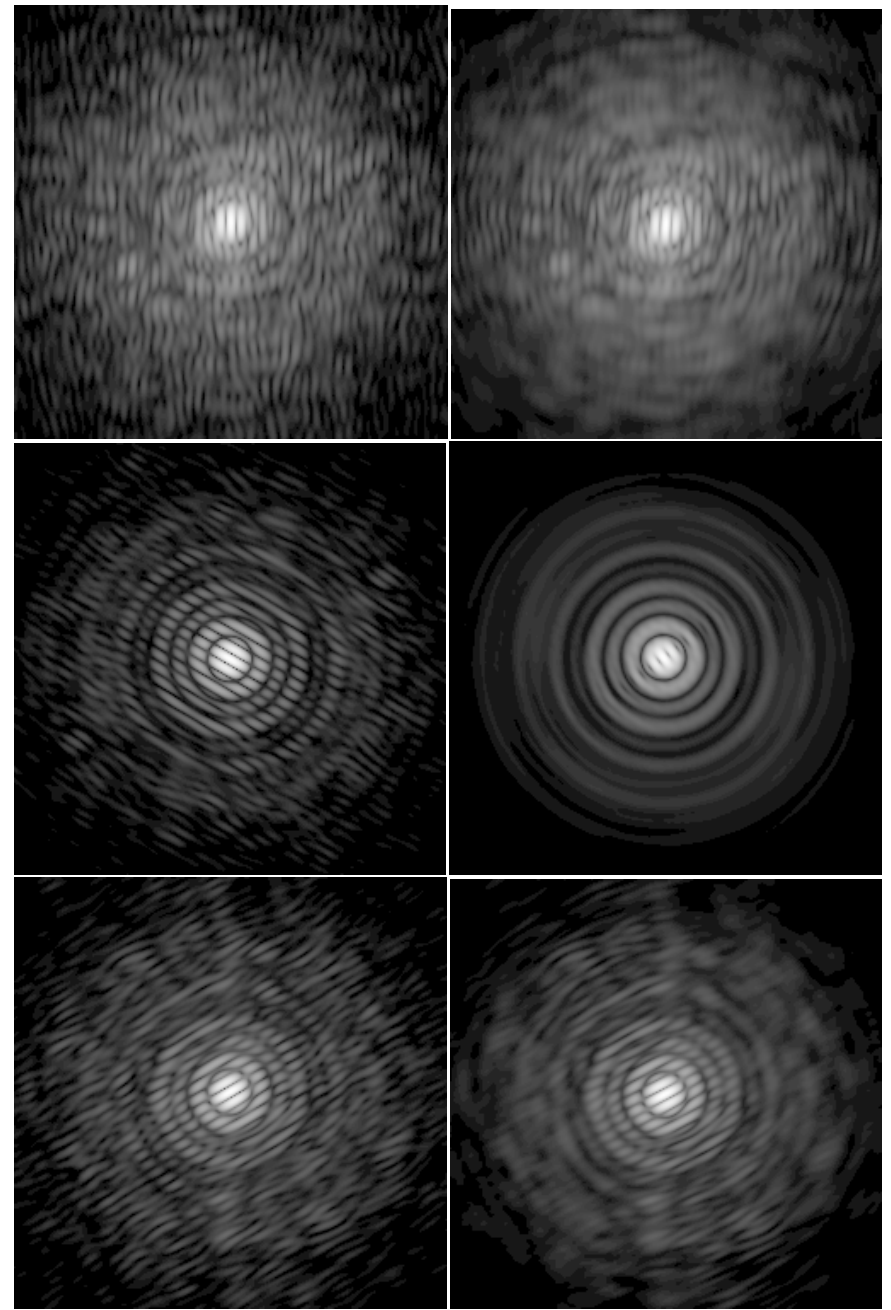

Fig. 3. The AO-corrected PSFs used in our simulations (logarithmic scale): in the left column the PSFs without angular smearing effect and in the right column those with angular smearing effect, for baseline orientations of $0^{\circ}, 60^{\circ}$ and $120^{\circ}$ (top to bottom).

one of these diffuse objects and was already used for the numerical simulations described in the first paper of this series (Correia et al. 2002), is moved to a distance of $1.4 \mathrm{kpc}$ instead of its actual $140 \mathrm{pc}$. All these objects are $128 \times 128$ pixels wide and correspond to an extension of $\sim 0$.' 85 (the pixel size is $6.67 \mathrm{mas}$ ).

Then we generate images by assuming ideal PSFs (corresponding to the three parallactic angles indicated above), an integration time of $20 \mathrm{~min}$, a sky background value of $12.5 \mathrm{mag} /{ }^{\prime \prime 2}$ ( $K$-band), a read-out noise (RON) of $2 \mathrm{e}^{-} \mathrm{rms}$, and a total transmission of $30 \%$. Moreover, for each of the diffuse objects we use three different magnitudes, arbitrarily fixed at 14, 15 and 16. An increase in magnitude is equivalent to a decrease of the SNR in the observed images. Therefore we consider 33 diffuse objects. The results of our experiments can be summarized as follows: for all the diffuse objects we have considered we can use $\omega=3$ if $m=14, \omega=5$ if $m=15$ and $\omega=8$ if $m=16$. As a rule, the acceleration exponent increases for decreasing SNR, so that when the SNR is low the algorithm can be very fast. Indeed, the reduction in the number of iterations with respect to OS-EM is just given by the acceleration exponent without modification of the restoration error. This is the minimum value of the relative rms error defined by:

$\rho_{\mathrm{rel}}^{(k)}=\frac{\left\|\boldsymbol{f}^{(k)}-\boldsymbol{f}\right\|}{\|\boldsymbol{f}\|}$,

where $\|$.$\| is the Euclidean norm, \boldsymbol{f}$ is the original object and $\boldsymbol{f}^{(k)}$ is the result of the $k$ th iteration. We have obtained an average restoration error of $9 \%$ for $m=14,10 \%$ for $m=15$ and $13 \%$ for $m=16$, with corresponding average numbers of iterations of 350,125 and 50. Without acceleration, the average numbers of iterations are, respectively, 1050, 625 and 400. However, the optimal number of iterations strongly depends on the specific object to be restored.

In a second set of experiments we perform a first comparison of the three methods OS-EM, RL-AV and RL-MM. To this purpose we fix the acceleration exponent at $\omega=2$ and we consider only one diffuse object, namely the YSO object IRAS $04302+2247$, already mentioned above, with magnitude 14. For each image we generate, by means of the software package AIRY, 50 noisy versions obtained with 50 different noise realizations (of Poisson type for the photon noise and of Gauss type for the RON). For each one of these realizations we increase the number of the iterations of the three methods up to reach the minimum of the restoration error (semiconvergence of the methods, see Bertero \& Boccacci 2000a), as defined in Eq. (19). Then, for each method, we compute the mean value $(M V)$ and the standard deviation $(S D)$ of the 50 values of the minimum restoration error and of the corresponding number of iterations. The results are reported in the first two rows of Table 1.

As follows from the first row, the most accurate restoration is provided by OS-EM while the two single-image methods are approximately equivalent. In all cases the standard deviation is quite small, and this means that the minimum restoration error does not depend strongly on the noise realization. On the other hand, the second row shows that RL-MM is the most efficient method, with also a good stability of the number of iterations with respect to variations of the noise realization. OS-EM is the less efficient one with also a rather broad dispersion of the optimal number of iterations.

However, since the minimum of the restoration error is, in general, rather flat, we have considered the number of iterations required for reaching the same accuracy with the three methods. We have chosen a threshold of $7 \%$ since this accuracy can be reached by all the methods. The results are reported in the third row and we can conclude that, from this point of view, OS-EM and RL-MM are equivalent.

In a third set of experiments we use three $256 \times 256$ versions of the same YSO object with different magnitudes ( $m=14$, 15 and 16). For each value of the magnitude three sets of images are generated by convolving the object with the three sets of PSFs described above and adding the noise contributions (sky background, photon noise, RON, and no dark current). In these experiments we consider only one noise realization, one for each set of PSFs. The purpose of these experiments is 
Table 1. Comparison between the accuracy and efficiency of the three methods. We have used a $128 \times 128$ image of the diffuse object IRAS 04302+2247 with magnitude $m=14$, convolved with ideal PSFs and corrupted with 50 different realizations of noise; the reported mean values $(M V)$ and standard deviations $(S D)$ refer to these 50 realizations.

\begin{tabular}{l|ll|ll|ll}
\hline \hline & \multicolumn{2}{|c|}{ OS-EM } & \multicolumn{2}{c|}{ RL-AV } & \multicolumn{2}{c}{ RL-MM } \\
& $M V$ & $S D$ & $M V$ & $S D$ & $M V$ & $S D$ \\
\hline Minimum of the restoration error (RE) & $4.8 \%$ & $\pm 0.1 \%$ & $6.6 \%$ & $\pm 0.1 \%$ & $6.2 \%$ & $\pm 0.1 \%$ \\
Optimal number of iterations & 1536 & \pm 141 & 705 & \pm 40 & 366 & \pm 13 \\
Number of iterations providing RE $\simeq 7 \%$ & 168 & \pm 6 & 385 & \pm 56 & 149 & \pm 6 \\
\hline
\end{tabular}

Table 2. Comparison between the accuracies of the three methods in the case of the diffuse object IRAS $04302+2247$ with different magnitudes; images $256 \times 256$ generated by means of ideal PSFs.

\begin{tabular}{l|lll}
\hline \hline Magnitude & OS-EM & RL-AV & RL-MM \\
\hline & \% error & $\%$ error & $\%$ error \\
14 & 6. & 7.3 & 7.2 \\
15 & 7.9 & 10 & 9.3 \\
16 & 9.8 & 11.4 & 12.6 \\
\hline
\end{tabular}

Table 3. Comparison between the accuracies of the three methods in the case of the diffuse object IRAS $04302+2247$ with different magnitudes; images $256 \times 256$ generated by means of AO-corrected PSFs.

\begin{tabular}{l|lll}
\hline \hline Magnitude & OS-EM & RL-AV & RL-MM \\
\hline & \% error & $\%$ error & $\%$ error \\
14 & 7.2 & 9.6 & 9.1 \\
15 & 8.6 & 11.1 & 10.2 \\
16 & 10 & 11.8 & 12.7 \\
\hline
\end{tabular}

Table 4. Comparison between the accuracies of the three methods in the case of the diffuse object IRAS 04302+2247 with different magnitudes; images $256 \times 256$ generated by means of AO-corrected PSF with angular smearing effect.

\begin{tabular}{l|lll}
\hline \hline Magnitude & OS-EM & RL-AV & RL-MM \\
\hline & \% error & \% error & \% error \\
14 & 7.4 & 10. & 8.7 \\
15 & 8.6 & 11.2 & 9.6 \\
16 & 10.2 & 12.8 & 11.6 \\
\hline
\end{tabular}

to compare the accuracy of the three methods and investigate its dependence on the magnitude of the object (hence on the SNR ratio) as well as on the AO-correction of the PSFs. The results are reported in Table 2 for the ideal PSFs, in Table 3 for the AO-corrected PSFs, and in Table 4 for the AO-corrected PSFs with the addition of the angular smearing effect. It follows that OS-EM always provides the best results even if the improvement of accuracy with respect to the other two methods does not strongly depend on the magnitude of the object and on the PSFs.

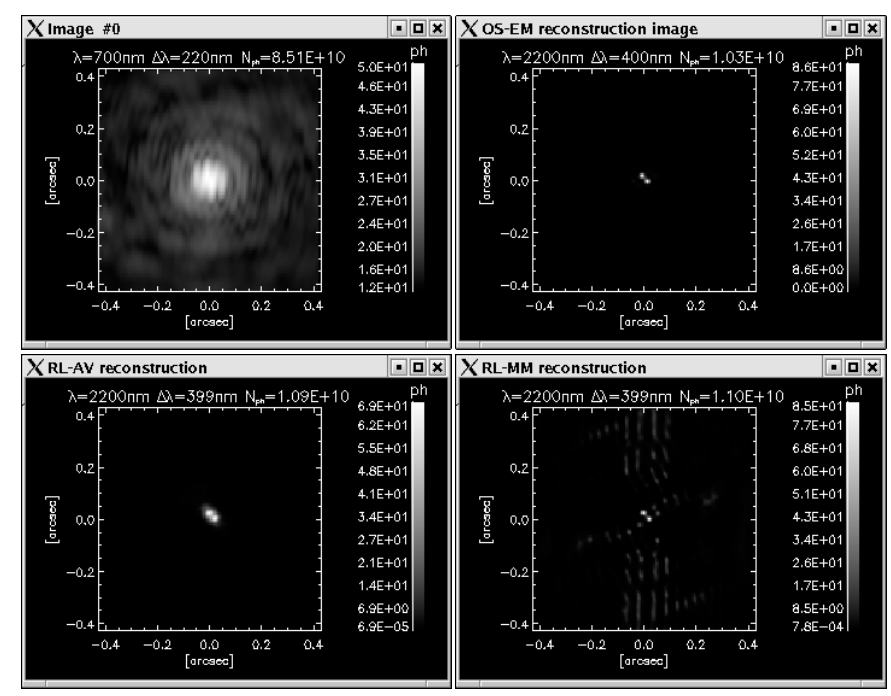

Fig. 4. Example of reconstruction of the binary system with magnitudes 10. The images have been obtained by means of the AO-corrected PSFs with angular smearing effect. In the upper panels, one of the three images and the reconstruction provided by OS$\mathrm{EM}$; in the lower panels, the reconstructions provided by RL-AV and RL-MM.

Finally, in a fourth set of experiments we consider the reconstruction of a binary system consisting of two stars with the same magnitude ( $m=10)$ and an angular separation which can be just resolved by LBT in $K$-band ( 25 mas). The purpose is to investigate the behaviour of the three methods for the three sets of PSFs. In Fig. 4 we give an example of the reconstructions we have obtained.

In the case of point objects the RL-like methods exhibit convergent behaviour (instead of the semiconvergent one for diffuse objects). This effect is shown in Fig. 5 where we plot the reconstructed value of the flux of the binary as a function of the number of iterations for the three sets of PSFs we have considered. The comparison between OS-EM and RL-MM needs some discussion.

As shown by the plots in the three panels, the OS-EM method always tends to underestimate the value of the flux, while in the first two cases RL-MM provides a small over-estimate. This effect is due to the setting zero of the negative values of the MM-image, given by Eq. (18), as we discussed in Sect. 3. As a consequence the total flux of the image is increased and this effect is at the origin 

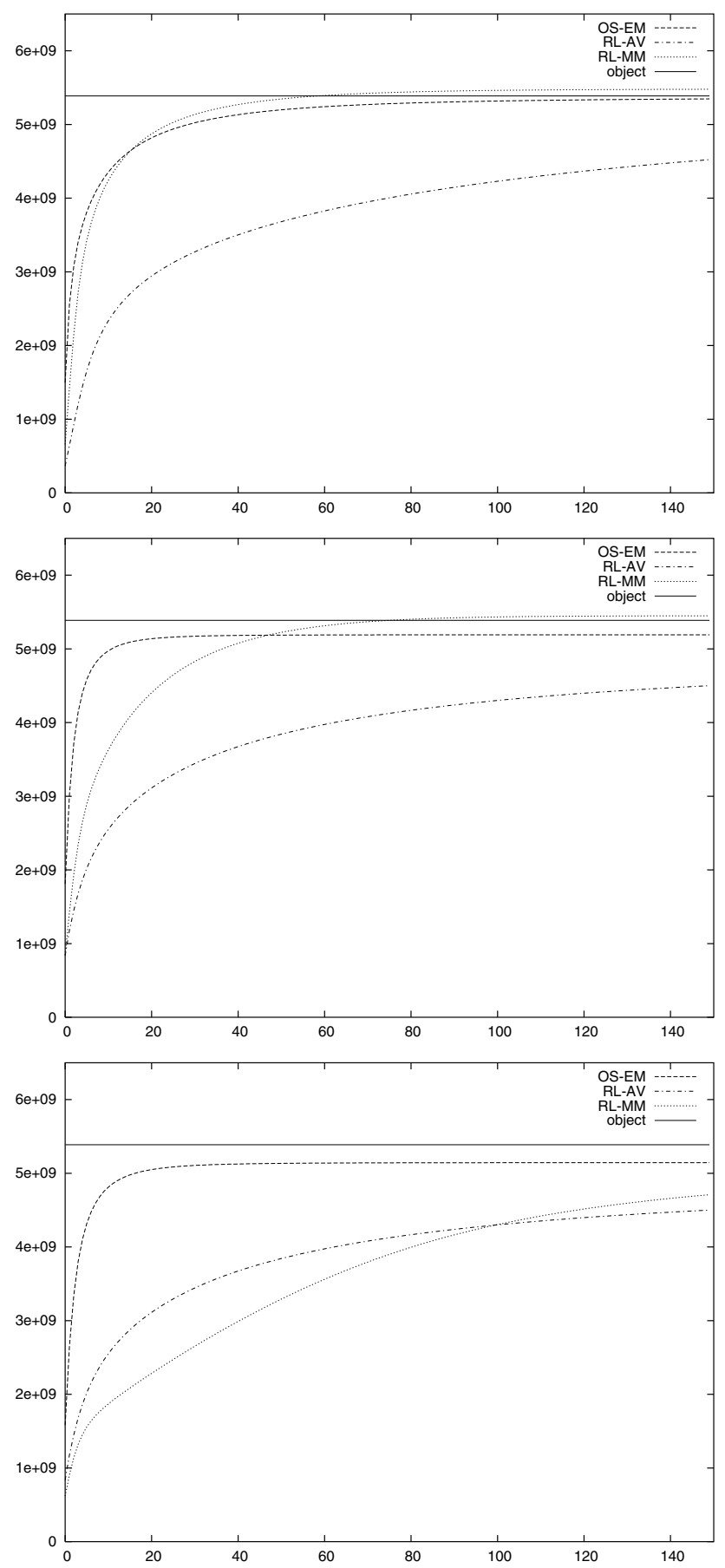

Fig. 5. Behaviour of the flux of the binary object, with magnitude 10, as a function of the number of iterations: ideal PSFs (upper panel); AO-corrected PSFs (middle panel): AO-corrected PSFs with angular smearing (lower panel).

of the over-estimation of the flux of the restored binary. In the third case (AO-corrected PSFs with angular smearing effect) OS-EM is definitely better than the other two methods.

\section{Concluding remarks}

In this paper we have investigated three different RL-like methods for efficiently reconstructing interferometric images provided by the LN beam combiner of LBT. In our opinion the accelerated version of the OS-EM method we propose in this paper is, at the moment, the best approach to a quick-look reconstruction of LN images with the same quality of the reconstruction provided by the RL method. The accelerated version of OS-EM may provide a speed-up of a factor 10 with respect to RL in the case of moderate SNR. However, it is obvious that further numerical experiments are required if one intends to provide the users with tables containing the acceleration factors as functions of the SNR.

We point out that an efficient and accurate quick-look method, providing nearly-real-time images when observing with LN will be of fundamental importance. Indeed the images obtained will have the unprecedented angular resolution of a nearly-23 m telescope, and hence will show previously unknown spatial features within the observed astrophysical objects.

\section{References}

Adorf, H. M., Hook, R. N., Lucy, L. B., \& Murtagh, F. D. 1993, ESO Conf. Proc., 41, 99

Anconelli, B., Bertero, M., Boccacci, P., \& Carbillet, M. 2005, A\&A, to be published

Bertero, M., \& Boccacci, P. 1998, Introduction to Inverse Problems in Imaging (Bristol: IOP Publishing)

Bertero, M., \& Boccacci, P. 2000a, A\&AS, 144, 181

Bertero, M., \& Boccacci, P. 2000b, A\&AS, 147, 323

Carbillet, M., Correia, S., Boccacci, P., \& Bertero, M. 2002, A\&A, 387,744

Carbillet, M., Vérinaud, C., Femenía, B., Riccardi, A., \& Fini, L. 2004, in preparation

Correia, S., Carbillet, M., Boccacci, P., Bertero, M., \& Fini, L. 2002, A\&A, 387, 733

Csiszár, I. 1991, Ann. Stat., 19, 2032

Holmes, T. J., \& Liu, Y.-H. 1991, JOSA, A8, 893

Hudson, H. M., \& Larkin, R. S. 1994, IEEE Trans. Med. Im., 13, 601

Kosarev, E. L., Peskov, V. D., \& Podolyak, E. R. 1983, Nucl. Instr. Meth., 208, 637

Lanteri, H., Roche, M., Cuevas, O., \& Aime, C. 2001, Signal Proc., 81,945

Lanteri, H., Roche, M., \& Aime, C. 2002, Inverse Problems, 18, 1397

Llacer, J., \& Nuñez, J. 1990, Proc. workshop, The Restoration of HST Images and Spectra - I, ed. R. L. White, \& R. J. Allen, 62

Lucy, L. 1974, AJ, 79, 745

Natterer, F., \& Wübbeling, F. 2001, Mathematical methods in Image Reconstruction (SIAM)

Richardson, W. H. 1972, JOSA, 62, 55

Snyder, D. L., Hammoud, A. M., \& White, R. L. 1993, JOSA, A10, 1014

Tanaka, I. 1987, IEEE Trans. Med. Im., MI-6, 98

Vio, R., Nagy, J., Tenorio, L., \& Wamsteker, W. 2004, A\&A, 416, 404 